\title{
Knockdown Resistance to DDT and Pyrethroids in the House Fly (Diptera: Muscidae): From Genetic Trait to Molecular Mechanism
}

\author{
DAVID M. SODERLUND AND DOUGLAS C. KNIPPLE
}

Department of Entomology, New York State Agricultural Experiment Station, Cornell University, Geneva, NY 14456

\begin{abstract}
Ann. Entomol. Soc. Am. 92(6): 909-915 (1999)
ABSTRACT Knockdown resistance to DDT and pyrethrins was the 1st insecticide resistance trait involving reduced target site sensitivity to be identified and isolated genetically. Almost 5 decades later, knockdown resistance continues to threaten the continued effectiveness of pyrethroid insecticides in the control of numerous agricultural pests and vectors of human disease. In this review we summarize progress in the characterization of knockdown resistance in the house fly, Musca domestica L., culminating in the identification of the specific sodium channel gene mutations that cause the reduced neuronal sensitivity to DDT and pyrethroids in knockdown-resistant insects.
\end{abstract}

KEY WORDS house fly, knockdown resistance, DDT, pyrethroids, genetic

SELECTION FOR INSECTICIDE resistance has been an undesirable by-product of insecticide use for almost a century (Forgash 1984). With the advent of inexpensive and highly effective insecticides after World War II, the rate of selection for resistance increased dramatically so that by $1990>500$ arthropod species were resistant to one or more insecticides or acaricides (Georghiou 1990). Today, the anticipation and strategic management of resistance is an integral component of the introduction of any new insect control agent.

Most insecticide resistance mechanisms fall into 2 broad categories: those involving enhanced metabolic detoxication and those involving reduced sensitivity of the insecticide target site (Soderlund and Bloomquist 1990, Soderlund 1997). Of these, the latter are particularly problematic because they often negate the effectiveness of entire classes of insecticides. Knockdown resistance to DDT and pyrethroids, the 1st target site resistance mechanism to be indentified, remains an important resistance mechanism that limits or threatens to limit the effectiveness of modern pyrethroid insecticides in many pest species. Knockdown resistance has remained a topic of continuing research interest and effort for $>4$ decades, but only recently has the molecular basis for this type of resistance been elucidated. In this special issue recognizing Carl W. Schaefer's 25-yr tenure as Editor of the Annals of the Entomological Society of America, it is particularly appropriate to summarize the progress in research over this 25 -yr span that has led from the preliminary toxicological, physiological, and genetic characterization of knockdown resistance in the house fly to a definitive description of the mechanism of knockdown resistance at the molecular level.

\section{Discovery}

Knockdown resistance $(k d r)$ in the house fly, Musca domestica L., was first described by Busvine (1951). These pioneering studies documented the existence in a field-derived strain from Italy of a factor that conferred resistance to the initial paralytic effects of DDT, DDT analogs, and pyrethrins but not to chlorinated cyclodienes. Moreover, knockdown resistance in the Italian strain was not correlated with metabolic dehydrochlorination of DDT, a mechanism known at that time to cause DDT resistance in other house fly strains (Busvine 1951, 1953). Subsequently, Milani (1954) and Milani and Travaglino (1957) isolated the $k d r$ resistance mechanism genetically from other DDT resistance traits and showed that it was caused by a recessive factor located on chromosome 3 .

The cross-resistance of $k d r$ flies to pyrethrins was confirmed in laboratory selection experiments. Busvine (1953) noted that further selection of the knockdown-resistant Italian strain with DDT produced a concurrent increase in the resistance of these flies to pyrethrins. Fine (1961) selected a house fly strain from Sudan with DDT and found that the resulting population exhibited increased resistance to DDT and pyrethrins. This study also documented the cross-resistance of DDT-selected flies to several early examples of pyrethroids, thereby providing evidence that the $k d r$ trait conferred cross-resistance to pyrethroids as a class rather than solely to the natural pyrethrins.

Recognition of the unique properties of the $k d r$ trait led researchers to search for similar traits in other DDT- and pyrethroid-resistant house fly strains. These efforts identified several additional resistance factors conferring $k d r$-like resistance (Tsukamoto et al. 1965, Plapp and Hoyer 1968, Farnham 1973, Scott and Georghiou 1986), which were recognized by their 
effect on knockdown, their unique cross-resistance spectra, and the failure of inhibitors of biotransformation to reduce the observed level of resistance. Research with European house fly populations exhibiting high levels of pyrethroid resistance led to the isolation of 2 pharmacologically distict alleles of a related trait, named super-kdr (Farnham et al. 1987, Sawicki 1978), which was also located on chromosome 3 . In contrast to the $k d r$ trait, which affords approximately equivalent protection to a wide variety of pyrethroids, the super-kdr trait confers particularly high levels of resistance to a subset of potent pyrethroid insecticides, such as deltamethrin, cypermethrin, and fenvalerate, that contain the $\alpha$-cyano-3-phenoxybenzyl alcohol moiety (Sawicki 1978, Farnham et al. 1987, Farnham and Khambay 1995).

Physiological and Pharmacological Characterization. In 1965, Tsukamoto et al. (1965) provided the 1st experimental evidence that a knockdown resistance factor localized to chromosome 3 greatly reduced the sensitivity of the house fly central nervous system to DDT. Since that time, electrophysiological assays, employing a variety of preparations from larval and adult $k d r$ insects, have confirmed that reduced neuronal sensitivity is the basis of the $k d r$ trait (Miller et al. 1979; Osborne and Hart 1979; Osborne and Smallcombe 1983; Pepper and Osborne 1993; Salgado et al. 1983; Scott and Georghiou 1986; Gibson et al. 1988, 1990). These studies documented that the reduction in neuronal sensitivity afforded by the $k d r$ trait is expressed in the fly nervous system throughout development and in both central and peripheral elements. Although the nervous system of super- $k d r$ insects also exhibits reduced neuronal sensitivity to pyrethroids in physiological assays, evidence that the super- $k d r$ trait confers a greater degree of resistance than $k d r$ at the level of the nerve is ambiguous and appears to depend on the test compound, nerve preparation and physiological endpoint employed as a basis for comparison (Nicholson et al. 1980; Gibson et al. 1988, 1990; Pepper and Osborne 1993).

Efforts to examine directly the binding of DDT or pyrethroids to their target site in nerve membrane preparations from susceptible and $k d r$ house flies have been hampered by the technical problems inherent in the use of lipophilic, low specific activity radioligands. Thus, studies of the binding of carbon-14 labeled DDT and cis-permethrin to house fly head membranes probably do not reflect the binding of these ligands to a toxicologically relevant site and do not provide insight into the mechanism underlying the $k d r$ trait (Chang and Plapp 1983). Recently, the pyrethroid binding site of rat brain sodium channels has been labeled stereospecifically with a novel pyrethroid radioligand (Trainer et al. 1997), but this approach has not yet been applied to studies of the pyrethroid binding site of insect sodium channels.

Knowledge that voltage-sensitive sodium channels are the primary targets for the action of DDT and pyrethroids (Sattelle and Yamamoto 1988, Soderlund and Bloomquist 1989, Bloomquist 1993, Narahashi 1996) has focused attention on the expression and pharmacology of house fly sodium channels in efforts to define the mechanism of knockdown resistance. Rossignol (1988) determined the apparent density of sodium channels (measured as binding sites for the sodium channel-specific radioligand $\left[{ }^{3} \mathrm{H}\right]$ saxitoxin) in head membranes prepared from adult susceptible and $k d r$ house flies. Results of these experiments showed that the sodium channel content of head membranes from $k d r$ flies was reduced by $>50 \%$, whereas the nicotinic acetylcholine receptor content, used as a marker for the yield of central nervous system tissue in these experiments, was unaltered. This finding suggested that reduced sodium channel expression was the mechanism of knockdown resistance. However, subsequent saxitoxin binding studies in other laboratories using multiple $k d r$ and super- $k d r$ strains failed to replicate this finding (Grubs et al. 1988, Sattelle et al. 1988, Pauron et al. 1989), and altered sodium channel expression in super-kdr house flies also was not detected by immunocytochemical techniques (Castella et al. 1997). The ability of the extent of sodium channel down-regulation observed by Rossignol (1988) to confer the magnitude of resistance afforded by the $k d r$ trait was also challenged on theoretical grounds (Grubs et al. 1988). Subsequent studies with a mutant strain of Drosophila melanogaster (Meigen) that exhibits constitutive down-regulation of sodium channel expression confirmed that a $50 \%$ reduction in sodium channel density is able to cause $k d r$-like resistance, but the magnitude of resistance resulting from a reduction in sodium channel expression of this magnitude is much less than that observed for $k d r$ house flies (Grubs et al. 1988, Kasbekar and Hall 1988).

The pharmacological properties of sodium channels have also been employed in indirect ways to explore the mechanism of knockdown resistance. Pyrethroids and DDT enhance allosterically the binding of $\left[{ }^{3} \mathrm{H}\right]$ batrachotoxinin A 20- $\alpha$-benzoate (BTX-B) to mammalian brain sodium channels (Brown et al. 1988, Lombet et al. 1988, Payne and Soderlund 1989, Rubin and Soderlund 1993). Extension of this approach to house fly head membranes documented a similar enhancement of BTX-B binding by the pyrethroid deltamethrin in 2 pyrethroid-susceptible strains but failed to detect any enhancement of binding in strains carrying the $k d r$ or super- $k d r$ resistance mechanism (Pauron et al. 1989). Other agents known to act on sodium channels have also been employed as pharmacological probes of the knockdown resistance mechanism. Physiological assays of motor nerve depolarization in house fly larval neuromuscular junction preparations showed that the resistance to pyrethroids conferred by the $k d r$ mechanism extended to the plant alkaloid aconitine (Salgado et al. 1983), which is known to act at the sodium channel at a binding site distinct from that for DDT and pyrethroids (Lombet et al. 1988). Paralysis bioassays using house fly larvae confirmed the resistance of $k d r$ insects to aconitine but did not find resistance to a variety of other compounds that act on sodium channels at several other distinct binding domains (Bloomquist and Miller 1986). Taken together, the results of these studies suggest that the $k d r$ 
mechanism involves a specific modification of the DDT/ pyrethroid sensitivity of house fly sodium channels with minimal effect on the remainder of the pharmacological profile of these channels.

A study of the lipid composition of nerve membranes in susceptible and resistant flies provided further insight into the knockdown resistance phenotype. Chiang and Devonshire (1982) measured the temperature-dependent catalytic activity of membrane-bound acetylcholinesterases in house fly head membrane preparations as a means of determining the transition temperatures of the nerve membranes of susceptible, $k d r$, and super- $k d r$ insects. Membranes from $k d r$ and super- $k d r$ insects exhibited higher membrane transition temperatures than those from susceptible insects, thereby implicating a role for alterations of the lipid composition of nerve membrane in the mechanism of knockdown resistance. However, these studies were unable to determine whether effects on the nerve membrane were a primary cause of resistance or a secondary and compensatory consequence of alterations in the functional properties of sodium channels in resistant insects.

\section{Genetic Linkage Analysis}

Farnham (1977) isolated house fly strains homozygous for 1 of 3 independently isolated knockdown resistance genes $(k d r, k d r$-NPR, and $k d r-O)$ and determined the crossover frequencies of each resistance gene with the markers bwb (brown body) and ge (green eye) located on chromosome 3. All 3 knockdown resistance genes exhibited similar crossover frequencies with each of the visible markers, and crosses between resistant strains showed that the 3 factors examined in this study are probably allelic. Although the super-kdr trait is widely presumed to be an allele of $k d r$ (Farnham et al. 1987, Sawicki 1978) there are no complementation data that explicitly support this conclusion.

More detailed genetic analyses of the $k d r$ and super$k d r$ traits were facilitated by studies that identified the product of the para (paralytic, a locus at which temperature-sensitive mutations impair nerve action potential conduction) gene of $D$. melanogaster as a physiologically important sodium channel (Loughney et al. 1989). Knowledge of the para sequence permitted the design of experiments to test the hypothesis that knockdown resistance is linked genetically to the house fly sodium channel gene that is orthologous to para. Oligonucleotide primers based on conserved regions of the para amino acid sequence were employed to amplify a small segment of the orthologous house fly gene (Knipple et al. 1991), which was used as a probe to isolate larger segments of this gene for use in genetic analyses.

Strain-specific restriction fragment-length polymorphism (RFLP) markers within the para-orthologous sodium channel gene of the house fly (designated $V s s c 1$ or $M s c$ ) were employed in combination with discriminating dose bioassays on individual house flies to assess the linkage between the Vsscl gene and the $k d r$ and super-kdr resistance traits (Williamson et al. 1993, Knipple et al. 1994). Results of these experiments demonstrated tight genetic linkage (within $\approx 1$ map unit) of both resistance traits and the Vsscl gene. In addition to providing strong genetic evidence for $\mathrm{mu}-$ tations at a sodium channel structural gene as the cause of knockdown resistance, these 2 studies also provided evidence for allelism of the $k d r$ and super- $k d r$ traits.

\section{Molecular Mechanism}

The genetic evidence implicating the Vsscl gene as the site of knockdown resistance mutations provided a strong impetus for the determination of the complete coding sequence of this gene (Ingles et al. 1996, Williamson et al. 1996). Comparison of partial and complete sequences from 15 house fly strains representing multiple examples of susceptible, $k d r$, and super-kdr phenotypes consistently identified 2 point mutations that were associated with resistant phenotypes: mutation of leucine to phenylalanine at amino acid residue 1014 (designated L1014F) in all $k d r$ and super- $k d r$ strains, and the additional mutation of methionine to threonine at residue 918 (designated M918T) only in super-kdr strains (Ingles et al. 1996, Miyazaki et al. 1996, Williamson et al. 1996). It is noteworthy that the M918T mutation has not been detected as a polymorphism in the absence of the L1014F mutation in any house fly population analyzed to date. This observation suggests that the M918T mutation produces channels with functional deficits that can be complemented, in part, by the L1014F mutation.

The identification of resistance-associated mutations does not, in itself, constitute evidence that such mutations are the cause of resistance. To complete the elucidation of the knockdown resistance mechanism, it was necessary to obtain the functional expression of sodium channels from susceptible insects and channels in which the resistance-associated mutations had been inserted by site-directed mutagenesis and to employ a suitable functional assay to assess the pyrethroid sensitivity of wildtype and specifically mutated channels. The unfertilized oocyte of the African clawed frog, Xenopus laevis, constitutes a powerful transient expression system for the study of a wide variety of neurotransmitter receptors and ion channels, including voltage-sensitive sodium channels from vertebrate nerve and muscle (Lester 1988, Catterall 1992). Recent studies showed that the Xenopus oocyte system effectively expresses $D$. melanogaster para and house fly Vssc1 sodium channels and that both the biophysical properties and the pyrethroid sensitivity of the expressed channels closely paralleled those of sodium channels in native neuronal preparations (Feng et al. 1995; Smith et al. 1997, 1998; Warmke et al. 1997; Lee et al. 1999b).

To test the functional significance of mutations associated with knockdown resistance, sodium channels encoded by cDNAs for the wildtype Vsscl sodium channel and specifically mutated channels containing the L1014F mutation associated with the $k d r$ pheno- 
type, the M918T/L1014 F double mutation associated with the super-kdr phenotype, and the M918T single mutation were expressed in Xenopus oocytes assayed for their sensitivity to the pyrethroid insecticides cismethrin and cypermethrin. The L1014F mutation decreased the sensitivity of house fly sodium channels to both cismethrin and cypermethrin by at least 10 -fold, which is sufficient to account for the degree of resistance to a wide structural variety of pyrethroids in $k d r$ insects (Smith et al. 1997). In contrast, the M918T/ L1014 double mutation greatly reduced the sensitivity of house fly sodium channels in this assay, so that no modification of sodium currents by either cismethrin or cypermethrin was detected at the maximum concentrations of these compounds attainable in this system (Lee et al. 1999b). The results of these experiments provide functional confirmation that the mutations associated with the $k d r$ and super-kdr phenotypes alter the sensitivity of house fly sodium channels to pyrethroids to an extent consistent with the degree of insecticide resistance conferred by these traits. These expression studies also assessed the effect on pyrethroid sensitivity of the M918T single mutation, which has not been found in fly populations except in combination with the L1014F mutation. Interestingly, expressed channels containing the M918T single mutation also appear to be completely insensitive to pyrethroids (Lee et al. 1999b).

\section{Conclusions and Implications}

The puzzle of knockdown resistance has, to a large extent, been solved. A single point mutation (L1014F) in the Vsscl sodium channel gene of the house fly is found in all knockdown-resistant strains that have been examined to date. Moreover, mutations corresponding to L1014F have been identified in the Vssc1orthologous sodium channel genes of pyrethroid-resistant strains of several other insect species (Miyazaki et al. 1996, Dong 1997, Guerrero et al. 1997, MartinezTorres et al. 1998, Schuler et al. 1998, Lee et al. 1999a). Functional expression experiments demonstrate that this single amino acid substitution, when inserted in the pyrethroid-susceptible wildtype sequence by sitedirected mutagenesis, reduces the sensitivity of house fly sodium channels sufficiently to account for the degree of resistance observed in toxicity bioassays with $k d r$ house fly strains.

These results not only identify the molecular mechanism of the $k d r$ trait but also provide important confirmation that sodium channels encoded by the Vssc1 gene are the principal target site for the toxic actions of DDT analogs and pyrethroids. Although the actions of DDT and pyrethroids on sodium channels have been characterized in great depth and detail over the past 3 decades, numerous other sites of action for some or all of these insecticides have also been proposed (Soderlund and Bloomquist 1989, Bloomquist 1993). The toxicological effect of the L1014F mutation in the $V s s c 1$ sodium channel implies that actions of DDT and pyrethroids at this target alone are sufficient to ac- count for the toxic effects of these compounds in whole insects.

The extension of this approach to the analysis of the super-kdr trait in the house fly has also clarified the relationship between the $k d r$ and super- $k d r$ traits. A 2nd amino acid substitution (M918T) was found in combination with the L1014F mutation in all super-kdr strains that have been examined, and the double mutation results in a virtual immunity to pyrethroids in expressed channels. Although the M918T mutation alone also confers a high level of pyrethroid resistance at the level of sodium channels expressed in oocytes, resistant populations with this single polymorphism apparently have not been selected in the field. The absence of such strains implies that the M918T mutation exerts a deleterious effect on sodium channel function in intact nerves that is rescued by the presence of the L1014F mutation. A further implication of this analysis is that the super-kdr trait did not arise independently but instead resulted from selection of a 2nd-site mutation in $k d r$ populations that already carried the L1014F mutation. This hypothesis has not been tested rigorously but is supported by RFLP analyses of the Vsscl locus in susceptible, $k d r$, and super$k d r$ house fly strains, which show strong conservation of RFLP patterns between several geographically distinct $k d r$ and super-kdr populations but extensive RFLP polymorphism among insecticide-susceptible strains (Williamson et al. 1995).

Although the L1014F mutation in the house fly and the corresponding mutation in the sodium channels of other insects is the most common polymorphism implicated in $k d r$-like resistance, several other resistance-associated mutations have also been identified in insect sodium channel gene sequences (Park et al. 1997, Pittendrigh et al. 1997, Head et al. 1998, Schuler et al. 1998). The multiplicity of sodium channel sequence polymorphisms associated with knockdown resistance contrasts with the situation for cyclodiene resistance in insects, which involves a mutation at a single amino acid residue in the subunit of the $\gamma$-aminobutyric acid receptor-chloride ionophore encoded by the $R d l$ gene in all cases that have been examined (french-Constant 1994). The diversity of sequence polymorphisms that may, in principle, be the cause of knockdown resistance poses a significant challenge for the use of this information in pyrethroid resistance monitoring and management. Although detection of the L1014F mutation in insect populations can provide strong circumstantial evidence for the existence of a knockdown resistance allele, the absence of this single polymorphism, or any other polymorphism previously shown to be associated with knockdown resistance, cannot be viewed as reliable evidence for the absence of knockdown resistance alleles.

\section{Acknowledgments}

We thank P. Adams, J. Bloomquist, K. Doyle, R. Grubs, P. Ingles, S. Lee, P. Marsella-Herrick, K. Nelson, and L. Payne for their contributions to research on knockdown resistance in our laboratories. These studies were supported in part by 
grants (89-37263-4425, 92-37302-7792, 94-37302-0408, and 9735302-4323) to the authors from the United States Department of Agriculture National Research Initiative Competitive Grants Program.

\section{References Cited}

Bloomquist, J. R. 1993. Neuroreceptor mechanisms in pyrethroid mode of action and resistance, pp. 181-226. In M. Roe and R. J. Kuhr [eds.], Reviews in pesticide toxicology, vol. 2. Toxicology Communications, Raleigh, NC.

Bloomquist, J. R., and T. A. Miller. 1986. Sodium channel neurotoxins as probes othe knockdown resistance mechanism. Neurotoxicology 7: 217-224.

Brown, G. B., J. E. Gaupp, and R. W. Olsen. 1988. Pyrethroid insecticides: stereospecific allosteric interaction with the batrachotoxinin-A benzoate binding site of mammalian voltage-sensitive sodium channels. Mol. Pharmacol. 34: 54-59.

Busvine, J. R. 1951. Mechanism of resistance to insecticide in houseflies. Nature (Lond.) 168: 193-195.

1953. Forms of insecticide-resistance in houseflies and body lice. Nature (Lond.) 171: 118-119.

Castella, C., N. Castells-Brooke, J.-B. Bergé, and D. Pauron. 1997. Expression and distribution of voltage-sensitive sodium channels in pyrethroid-susceptible and pyrethroidresistant Musca domestica. Invertebr. Neurosci. 3: 41-47.

Catterall, W. A. 1992. Cellular and molecular biology of voltage-gated sodium channels. Physiol. Rev. 72: S15-S48.

Chang, C. P., and F. W. Plapp, Jr. 1983. DDT and pyrethroids: receptor binding in relation to knockdown resistance $(k d r)$ in the house fly. Pestic. Biochem. Physiol. 20: 86-91.

Chiang, C., and A. L. Devonshire. 1982. Changes in membrane phospholipids, identified by Arrhenius plots of acetylcholinesterase and associated with pyrethroid resistance $(k d r)$ in houseflies (Musca domestica). Pestic. Sci. 13: $156-160$.

Dong, K. 1997. A single amino acid change in the para sodium channel protein is associated with knockdown-resistance $(k d r)$ to pyrethroid insecticides in the German cockroach. Insect Biochem. Mol. Biol. 27: 93-100.

Farnham, A. W. 1973. Genetics of resistance of pyrethroidselected houseflies, Musca domestica L. Pestic. Sci. 4: 513-520.

Farnham, A. W. 1977. Genetics of resistance of house flies (Musca domestica L.) to pyrethroids. I. Knockdown resistance. Pestic. Sci. 8: 631-636.

Farnham, A. W., A.W.A. Murray, R. M. Sawicki, I. Denholm, and J. C. White. 1987. Characterization of the structureactivity relationship of $k d r$ and two variants of super- $k d r$ to pyrethroids in the house fly (Musca domestica L.). Pestic. Sci. 19: 209-220.

Farnham, A. W., and B.P.S. Khambay. 1995. The pyrethrins and related compounds. Part XXXIX. Structure-activity relationships of pryethroidal esters with cyclic side chains in the alcohol component against resistant strains of housefly (Musca domestica). Pestic. Sci. 44: 269-275.

Feng, G., P. Deak, M. Chopra, and L. M. Hall. 1995. Cloning and functional analysis of TipE, a novel membrane protein that enhances Drosophila para sodium channel function. Cell 82: 1001-1011.

French-Constant, R. H. 1994. The molecular and population genetics of cyclodiene insecticide resistance. Insect Biochem. Mol. Biol. 24: 335-345.

Fine, B. C. 1961. Pattern of pyrethrin-resistance in houseflies. Nature (Lond.) 191: 884-885.
Forgash, A. J. 1984. History, evolution, and consequences of insecticide resistance. Pestic. Biochem. Physiol. 22: $178-$ 186.

Georghiou, G. P. 1990. Overview of insecticide resistance, pp. 18-41. In M. B. Green, H. M. LeBaron, and W. K. Moberg [eds.], Managing resistance to agrochemicals: from fundamental to practical strategies. American Chemical Society, Washington, DC.

Gibson, A. J., M. P. Osborne, H. F. Ross, and R. M. Sawicki. 1988. An electrophysiological investigation of the susceptible (Cooper) and resistant $(k d r$; super- $k d r)$ strains of the adult housefly, Musca domestica L. Pestic. Sci. 23: 283-292.

Gibson, A. J., M. P. Osborne, H. F. Ross, and R. M. Sawicki. 1990. An electrophysiological study of susceptible (Cooper) and resistant ( $k d r$; super- $k d r)$ strains of the adult housefly (Musca domestica L.) using an isolated metathoracic leg preparation. Pestic. Sci. 30: 379-396.

Grubs, R. E., P. M. Adams, and D. M. Soderlund. 1988. Binding of $\left[{ }^{3} \mathrm{H}\right]$ saxitoxin to head membrane preparations from susceptible and knockdown-resistant house flies. Pestic. Biochem. Physiol. 32: 217-223.

Guerrero, F. D., R. C. Jamroz, D. Kammlah, and S. E. Kunz. 1997. Toxicological and molecular characterization of pyrethroid-resistant horn flies, Haematobia irritans: identification of $k d r$ and super- $k d r$ point mutations. Insect Biochem. Mol. Biol. 27: 745-755.

Head, D. J., A. R. McCaffery, and A. Callaghan. 1998. Novel mutations in the para-homologous sodium channel gene associated with phenotypic expression of nerve insensitivity resistance to pyrethroids in Heliothine lepidoptera. Insect Mol. Biol. 7: 191-196.

Ingles, P. J., P. M. Adams, D. C. Knipple, and D. M. Soderlund. 1996. Characterization of voltage-sensitive sodium channel gene coding sequences from insecticidesusceptible and knockdown-resistant house fly strains. Insect Biochem. Mol. Biol. 26: 319-326.

Kasbekar, D. P., and L. M. Hall. 1988. A Drosophila mutation that reduces sodium channel number confers resistance to pyrethroid insecticides. Pestic. Biochem. Physiol. 32: 135-145.

Knipple, D. C., L. L. Payne, and D. M. Soderlund. 1991. PCR-generated conspecific sodium channel gene probe for the house fly homologue of the para locus of Drosophila melanogaster. Arch. Insect Biochem. Physiol. 16: $45-53$.

Knipple, D. C., K. E. Doyle, P. A. Marsella-Herrick, and D. M. Soderlund. 1994. Tight genetic linkage between the $k d r$ insecticide resistance trait and a voltage-sensitive sodium channel gene in the house fly. Proc. Natl. Acad. Sci. U.S.A. 91: 2483-2487.

Lee, S. H., J. B. Dunn, J. M. Clark, and D. M. Soderlund. 1999a. Molecular analysis of $k d r$-like resistance in a permethrin-resistant strain of Colorado potato beetle. Pestic. Biochem. Physiol. 63: 63-75.

Lee, S. H., T. J. Smith, D. C. Knipple, and D. M. Soderlund. 1999b. Mutations in the house fly Vssc1 sodium channel gene associated with super- $k d r$ resistance abolish the pyrethroid sensitivity of Vsscl/tipE sodium channels expressed in Xenopus oocytes. Insect Biochem. Mol. Biol. 29: $185-194$.

Lester, H. A. 1988. Heterologous expression of excitability proteins: route to more specific drugs? Science (Wash. D.C.) $241: 1057-1063$.

Lombet, A., C. Mourre, and M. Lazdunski. 1988. Interactions of insecticides of the pyrethroid family with specific binding sites on the voltage-dependent sodium channel from mammalian brain. Brain Res. 459: 44-53. 
Loughney, K., R. Kreber, and B. Ganetzky. 1989. Molecular analysis of the para locus, a sodium channel gene in Drosophila. Cell 58: 1143-1154.

Martinez-Torres, D., F. Chandre, M. S. Williamson, F. Darriet, J. B. Bergé, A. L. Devonshire, P. Guillet, N. Pasteur, and D. Pauron. 1998. Molecular characterization of pyrethroid knockdown resistance $(k d r)$ in the major malaria vector Anopheles gambiae s.s. Insect Mol. Biol. 7: 179-184.

Milani, R. 1954. Comportamento mendeliano della resistenza alla azione abbattante del DDT: correlazione tran abbattimento e mortalita in Musca domestica L. Riv. Parassitol. 15: 513-542.

Milani, R., and A. Travaglino. 1957. Richerche genetische sulla resistenza al DDT in Musca domestica concateazione del gene $k d r$ (knockdown-resistance) con due mutanti morphologigi. Riv. Parassitol. 18: 199-202.

Miller, T. A., J. M. Kennedy, and C. Collins. 1979. CNS insensitivity to pyrethroids in the resistant $k d r$ strain of house flies. Pestic. Biochem. Physiol. 12: 224-230.

Miyazaki, M., K. Ohyama, D. Y. Dunlap, and F. Matsumura. 1996. Cloning and sequencing of the para-type sodium channel gene from susceptible and $k d r$-resistant German cockroaches (Blattella germanica) and house fly (Musca domestica). Mol. Gen. Genet. 252: 61-68.

Narahashi, T. 1996. Neuronal ion channels as the target sites of insecticides. Pharmacol. Toxicol. 78: 1-14.

Nicholson, R. A., R. J. Hart, and M. P. Osborne. 1980. Mechanisms involved in the development of resistance to pyrethroids with particular reference to knockdown resistance in houseflies, pp. 465-471. In Insect neurobiology and pesticide action. Society of Chemical Industry, London.

Osborne, M. P., and R. J. Hart. 1979. Neurophysiological studies of the effects of permethrin upon pyrethroid resistant $(k d r)$ and susceptible strains of dipteran larvae. Pestic. Sci. 10: 407-413.

Osborne, M. P., and A. Smallcombe. 1983. Site of action of pyrethroid insecticides in neuronal membranes as revealed by the $\mathrm{kdr}$ resistance factor, pp. 103-107. In J. Miyamoto and P. C. Kearney [eds.], Pesticide Chemistry: Human Welfare and the Environment, vol. 3. Pergamon, New York.

Park, Y., M.F.J. Taylor, and R. Feyereisen. 1997. A valine 421 to methionine mutation in IS6 of the $h s c p$ voltage-gated sodium channel associated with pyrethroid resistance in Heliothis virescens F. Biochem. Biophys. Res. Commun. 239: 688-691.

Pauron, D., J. Barhanin, M. Amichot, M. Pralavorio, J.-B. Berge, and M. Lazdunski. 1989. Pyrethroid receptor in the insect $\mathrm{Na}^{+}$channel: alteration of its properties in pyrethroid-resistant flies. Biochemistry 28: 1673-1677.

Payne, G. T., and D. M. Soderlund. 1989. Allosteric enhancement by DDT of the binding of $\left[{ }^{3} \mathrm{H}\right]$ batrachotoxinin A-20- $\alpha$-benzoate to sodium channels. Pestic. Biochem. Physiol. 33: 276-282.

Pepper, D. R., and M. P. Osborne. 1993. Electrophysiological identification of site-insensitive mechanisms in knockdown-resistant strains $(k d r$, super- $k d r)$ of the housefly larva (Musca domestica). Pestic. Sci. 39: 279-286.

Pittendrigh, B., R. Reenan, R. H. ffrench-Constant, and B. Ganetzky. 1997. Point mutations in the Drosophila sodium channel gene para associated with resistance to DDT and pyrethroid insecticides. Mol. Gen. Genet. 256: 602-610.

Plapp, F. W., Jr., and R. F. Hoyer. 1968. Possible pleiotropism of a gene conferring resistance to DDT, DDT analogs and pyrethrins in the housefly and Culex tarsalis. J. Econ. Entomol. 61: 761-765.

Rossignol, D. P. 1988. Reduction in number of nerve membrane sodium channels in pyrethroid resistant house flies. Pestic. Biochem. Physiol. 32: 146-152.

Rubin, J. G., and D. M. Soderlund. 1993. Structure-activity relationships for pyrethroids and DDT analogs as modifiers of $\left[{ }^{3} \mathrm{H}\right]$ batrachotoxinin A 20 - $\alpha$-benzoate binding to mouse brain sodium channels. Pestic. Biochem. Physiol. 45: $130-140$.

Salgado, V. L., S. N. Irving, and T. A. Miller. 1983. Depolarization of motor nerve terminals by pyrethroids in susceptible and $k d r$-resistant house flies. Pestic. Biochem. Physiol. 20: 100-114.

Sattelle, D. B., C. A. Leech, S.C.R. Lummis, B. J. Harrison, H.P.C. Robinson, G. D. Moores, and A. L. Devonshire. 1988. Ion channel properties of insects susceptible and resistant to insecticides, pp. 563-582. In G. G. Lunt [ed.], Neurotox '88: the molecular basis of drug and pesticide action. Elsevier, Amsterdam.

Sattelle, D. B., and D. Yamamoto. 1988. Molecular targets of pyrethroid insecticides. Adv. Insect Physiol. 20: 147-213.

Sawicki, R. M. 1978. Unusual response of DDT-resistant houseflies to carbinol analogues of DDT. Nature (Lond.) 275: 443-444.

Schuler, T. H., D. Martinez-Torres, A. J. Thompson, I. Denholm, A. L. Devonshire, I. R. Duce, and M. S. Williamson. 1998. Toxicological, electrophysiological, and molecular characterisation of knockdown resistance to pyrethroid insecticides in the diamondback moth, Plutella xylostella (L.). Pestic. Biochem. Physiol. 59: 169-192.

Scott, J. G., and G. P. Georghiou. 1986. Mechanisms responsible for high levels of permethrin resistance in the house fly. Pestic. Sci. 17: 195-206.

Smith, T. J., P. J. Ingles, and D. M. Soderlund. 1998. Actions of the pyrethroid insecticides cismethrin and cypermethrin on house fly Vssc1 sodium channels expressed in Xenopus oocytes. Arch. Insect Biochem. Physiol. 38: 126 136.

Smith, T. J., S. H. Lee, P. J. Ingles, D. C. Knipple, and D. M. Soderlund. 1997. The L1014F point mutation in the house fly Vssc1 sodium channel confers knockdown resistance to pyrethroids. Insect Biochem. Mol. Biol. 27: 807-812.

Soderlund, D. M. 1997. Molecular mechanisms of insecticide resistance, pp. 21-56. In V. Sjut [ed.], Molecular mechanisms of resistance to agrochemicals. Springer, Berlin.

Soderlund, D. M., and J. R. Bloomquist. 1989. Neurotoxic actions of pyrethroid insecticides. Annu. Rev. Entomol. 34: 77-96.

Soderlund, D. M., and J. R. Bloomquist. 1990. Molecular mechanisms of insecticide resistance, pp. 58-96. In R. T. Roush and B. E. Tabashnik [eds.], Pesticide resistance in arthropods. Chapman \& Hall, New York.

Trainer, V. L., J. C. McPhee, H. Boutelet-Bochan, C. Baker, T. Scheuer, D. Babin, J.-P. Demoute, D. Guedin, and W. A. Catterall. 1997. High affinity binding of pyrethroids to the $\alpha$ subunit of brain sodium channels. Mol. Pharmacol. 51: 651-657.

Tsukamoto, M., T. Narahashi, and T. Yamasaki. 1965. Genetic control of low nerve sensitivity to DDT in insecticide-resistant houseflies. Botyu-Kagaku 30: 128-132.

Warmke, J. W., R.A.G. Reenan, P. Wang, S. Qian, J. P. Arena, J. Wang, D. Wunderler, K. Liu, G. J. Kaczorowski, L.H.T. Van der Ploeg, and others. 1997. Functional expression of Drosophila para sodium channels: modulation by the 
membrane protein tipE and toxin pharmacology. J. Gen. Physiol. 110: 119-133.

Williamson, M. S., C. A. Bell, I. Denholm, and A. L. Devonshire. 1995. Analysis of sodium channel gene sequences from susceptible and knockdown resistant $(k d r)$ houseflies, Musca domestica, pp. 86-96. In J. M. Clark [ed.], Molecular action and pharmacology of insecticides on Ion channels. American Chemical Society, Washington, DC.

Williamson, M. S., I. Denholm, C. A. Bell, and A. L. Devonshire. 1993. Knockdown resistance $(k d r)$ to DDT and pyrethroid insecticides maps to a sodium channel gene locus in the housefly (Musca domestica). Mol. Gen. Genet. 240: 17-22.

Williamson, M. S., D. Martinez-Torres, C. A. Hick, and A. L. Devonshire. 1996. Identification of mutations in the housefly para-type sodium channel gene associated with knockdown resistance $(k d r)$ to pyrethroid insecticides. Mol. Gen. Genet. 252: 51-60.

Received for publication 20 November 1998; accepted 18 February 1999. 